\title{
Coulisses
}

Revue de théâtre

40 | Hiver 2010

Jeux de rappels chez Marivaux

\section{Deux peintres : Fra Filippo Lippi et Andrea del Sarto}

Deux monologues traduits très librement de l'anglais de Robert Browning

Robert Browning et David Ball

\section{(2) OpenEdition}

12 Journals

Édition électronique

URL : https://journals.openedition.org/coulisses/665

DOI : 10.4000/coulisses. 665

ISSN : 2546-9460

Éditeur

Presses universitaires de Franche-Comté

Édition imprimée

Date de publication : 1 janvier 2010

Pagination : 119-133

ISBN : 978-2-84867-283-0

ISSN : $1150-594 \mathrm{X}$

\section{Référence électronique}

Robert Browning et David Ball, « Deux peintres : Fra Filippo Lippi et Andrea del Sarto », Coulisses [En ligne], 40 | Hiver 2010, mis en ligne le 30 novembre 2016, consulté le 29 décembre 2022. URL : http:// journals.openedition.org/coulisses/665; DOI : https://doi.org/10.4000/coulisses.665

Ce document a été généré automatiquement le 29 décembre 2022.

Tous droits réservés 


\title{
Deux peintres : Fra Filippo Lippi et Andrea del Sarto
}

\author{
Deux monologues traduits très librement de l'anglais de Robert \\ Browning
}

\author{
Robert Browning et David Ball
}

1 Les monologues dramatiques de Browning, écrits pour la plupart dans les années 1850 et 1860 , occupent une position charnière dans la tradition de la poésie anglaise: entre une poésie romantique toujours influencée par le grand style noble de Milton, et la poésie moderniste en vers libres du début du vingtième siècle, influencée par l'œuvre de Rimbaud, de Corbière et de Laforgue et par les rythmes de la langue parlée. Ces monologues ont une qualité indéniablement dramatique: une voix, un personnage, une histoire, bien individualisés, mais permettant aussi, de manière distante, indirecte, déguisée, à la voix de Browning lui-même, en sage victorien, de se faire entendre.

2 Cependant, comme la plupart de la poésie écrite en Europe depuis la Renaissance, ils sont destinés à la lecture et à la relecture, solitaire et silencieuse. Ils sont très peu représentables et, en effet, je n'ai jamais entendu parler de leur présentation en spectacle. Leur langage est souvent trop dense, ambigu même, et les structures grammaticales trop compliquées, pour être assez facilement compréhensibles de la part de l'auditeur qui les entendrait sur scène pour la première fois.

D'où l'occasion en or offerte au traducteur ! Car, traduits dans une autre langue, contemporaine et accessible, ils deviennent représentables. Ma traduction est ainsi très libre, plutôt une adaptation, mettant l'accent dès le départ sur la théâtralité. Et pour que le lecteur français ait ici quelque possibilité de faire son propre jugement sur la fidélité et l'infidélité de l'adaptation, nous mettons le début de chaque poème en anglais face au français.

Les deux monologues vont naturellement ensemble, les deux qui sont consacrés à des peintres de la Renaissance italienne. Browning était lui-même un passionné de l'Italie, d'une Italie mythique, celle des gloires de la Renaissance. Et ce fut à Florence qu'il passa les années sans doute les plus heureuses et les plus fécondes de sa vie, 1846-61, celles de son mariage avec la poétesse, Elizabeth Barrett Browning. 
5 Le thème du premier monologue est une défense de ce que nous appellerions aujourd'hui le réalisme, le respect de la vérité de la nature, un respect moral et pratique aussi bien qu'artistique, tandis que le deuxième esquisse une réponse typiquement victorienne à la question: un art grand et noble, exige-t-il une grandeur d'âme correspondante de la part de l'artiste? Le peintre Andrea del Sarto se demande pourquoi il n'a pas fait si bien que Raphaël et Michel-Ange, bien que sa compétence technique soit égale sinon supérieure à la leur...

\section{Fra Lippo Lippi (Robert Browning)}

I am poor brother Lippo, by your leave! You need not clap your torches to my face. Zooks, what's to blame? you think you see a monk! What, it's past midnight, and you go the rounds, And here you catch me at an alley's end Where sportive ladies leave their doors ajar. The Carmine's my cloister: hunt it up, Do, - harry out, if you must show your zeal, Whatever rat, there, haps on his wrong hole, And nip each softling of a wee white mouse, Weke, weke, that's crept to keep him company! Aha, you know your betters? Then, you'll take Your hand away that's fiddling on my throat, And please to know me likewise. Who am I? Why, one, sir, who is lodging with a friend Three streets off - he's a certain... how d'ye call? Master - a... Cosimo of the Medici, In the house that caps the corner. Boh! you were best! Remember and tell me, the day you're hanged, How you affected such a gullet's-gripe! But you, sir, it concerns you that your knaves Pick up a manner nor discredit you. Zooks, are we pilchards, that they sweep the streets And count fair prize what comes into their net? He's Judas to a tittle, that man is! Just such a face! why, sir, you make amends. Lord! I'm not angry! Bid your hangdogs go Drink out this quarter-florin to the health of the munificent House that harbours me (And many more beside, lads! more beside!) And all's come square again. I'd like his face His, elbowing on his comrade in the door With the pike and lantern, - for the slave that holds John Baptist's head a-dangle by the hair.

\section{Fra Filippo Lippi (David Ball)}

La nuit, vers l'aube. Une rue de Florence. Le moine peintre, en train de rentrer discrètement, est arrêté sans ménagement par le guet. Au metteur en scène de décider de combien d'hommes sera composé le guet, si l'un d'eux en sera le chef ou s'ils seront tous à égalité, et comment seront réparties leurs répliques.

LE GUET : Halte ! Qui va là ? 
FILIPPO LIPPI : Doucement, mes amis! Je ne suis qu'un pauvre frère, le frère Filippo, à votre service. Pas besoin de me prendre comme ça. Vous êtes étonnés, n'est-ce pas? De voir un moine dehors à cette heure-ci.

LE GUET : Et dans cette rue surtout.

FILIPPO : Une rue mal famée. Et pourquoi donc? Parce que s'y trouvent de charmantes dames qui laissent leurs portes entrouvertes? Mon couvent, sachez-le, est le Carmine. Allez-y, si vous êtes si pleins de zèle, et chassez tous les rats là-bas qui se sont trompés de trou, et toutes les petites souris blanches qui leur tiennent compagnie. Non, vous n'osez pas? Lâchez-moi, donc. Savez-vous d'où je viens? Cosimo de Médicis, ce nom vous dit quelque chose... Son palais n'est pas loin, n'est-ce pas? Vous n'avez pas envie, je suppose, de finir sur l'échafaud, une corde autour du cou? Non, bien sûr que non. Vous n'êtes pas de ces pêcheurs qui attrapent tout, bêtement, dans leurs filets. Toi, mon vieux, avec ton visage, tu pourrais prendre le rôle de Judas sans le moindre déguisement, je t'assure, celui qui a trahi le Christ.

LE GUET : Nos excuses, mon frère, nous nous sommes trompés.

FILIPPO : Bon, d'accord, je ne suis pas fâché. Tenez, voici une pièce à partager, pour boire à ma santé et à celle du palais. Nous sommes nombreux, vous savez, dans ce havre du mécénat.

LE GUET : Merci, mon frère.

FILIPPO : Ton visage à toi, par contre, serait parfait pour l'esclave qui tient la tête de St Jean Baptiste. Voyez, il tient la tête dans une main, par les cheveux, et dans l'autre, son épée, encore ensanglantée. Si vous aviez par hasard, dans votre poche, un morceau de craie, ou de fusain, je pourrais vous faire voir. Je suis peintre, vous comprenez.

LE GUET : Frère Filippo le peintre ! Bien sûr que nous avons entendu parler de vous.

FILIPPO : Mais oui, je suis connu. Et si on passait un petit moment ensemble, messieurs, à causer... Il y a du printemps dans l'air, n'est-ce pas ? C'est la saison des carnavals, des fêtes, qui plaisent tant aux jeunes qui aiment chanter et courir par les rues. Ça fait trois semaines que je suis enfermé chez le prince, à peindre, des saints, des saintes. Mais la nuit, je ne peux pas peindre. Alors, j'ai tout simplement ouvert la fenêtre, pour prendre un peu d'air frais, et juste à ce moment-là il y avait une bande de jeunes qui passait. Ils dansaient, chantaient. (Il chante.) «Sans l'amour, la terre n'est qu'une tombe. Je suis tout seul : j'ai laissé partir ma blonde ! Il y avait des filles. Il y en avait une qui m'a vu, à la fenêtre, et son regard! Ses yeux, ses petits yeux brillants! Mes amis, je ne suis qu'un homme! Mais comment descendre? Voici ce que j'ai fait. Les draps, les rideaux, le jeté de lit, tout, j'ai tout pris, j'ai tout noué, et je me suis fait une échelle. Pas tout à fait assez longue, tant pis, j'ai dû me laisser tomber le dernier bout. Sans mal, heureusement. Et je les ai rattrapés. Ils m'ont accueilli, en chantant : « Ah, ma fleur, ma fée, si j'ai été heureux, que m'importe qui le sait ? » Eh bien, -

LE GUET : Eh bien, oui, mon frère?

FILIPPO : Eh bien, j'étais en train de rentrer tout doucement au palais quand vous m'avez attrapé là comme dans un piège !

LE GUET : Toutes nos excuses, mon frère, encore une fois.

FILIPPO : J'ai un St Jérôme à faire demain. Celui qui se frappait sur la poitrine avec une pierre pour mater les tentations de la chair ! Et vous croyez que je n'ai pas besoin d'un peu de repos, d'un peu de sommeil, avant de m'y attaquer? Non, je le vois bien, vous êtes toujours choqués que je sois un moine. Si vous étiez tombés sur le prince Cosimo, vous n'auriez rien dit. Mais moi! Bon, parlons-en un peu, de moi, si vous voulez bien. Je 
vais vous expliquer.

Ma mère est morte quand je n'étais qu'un bébé, mon père aussi. Je n'avais personne, j'étais dans la rue. Tout ce que j'ai mangé, pendant plus d'un an, c'étaient des pelures de fruits, des écorces de melons. Et puis un matin, il gelait, il y avait du vent aussi, j'étais plié en deux de froid, de faim, et je suis tombé, comme ça, par terre. Alors, une vieille dame m'a trouvé, elle m'a pris par la main, et elle m'a emmené jusqu'à la porte du couvent, où quelqu'un m'a donné un bout de pain, le premier que j'ai eu à manger ce mois-là. Et le prieur, un gros bonhomme, gentil, il était en train de manger quelque chose lui aussi, m'a dit, il s'est essuyé la bouche et m'a dit : Alors, mon petit, tu es prêt à quitter ce monde ? A renoncer - Et moi, je me suis dit, à renoncer à ce bout de pain ? Nenni! Bref, ils m'ont fait moine. J'ai renoncé au monde et à toutes ses vanités, aux palais, aux fermes, aux banques, aux commerces. J'ai renoncé à tout ce que les Médicis, par exemple, ont à cœur. Et à l'âge de huit ans, messieurs! C'est à huit ans que j'ai fait cela!

Mais manger à sa faim, être au chaud, paresser toute la journée à ne rien faire, j'ai découvert plus tard, bien sûr, qu'il y avait un prix à payer pour tout cela. Qu'est-ce qu'on peut faire de ce garçon? C'était ça la question. Un copiste? Ils ont essayé de m'apprendre le latin. Ça n'a pas marché. Le seul mot que j'ai retenu, je crois, c'est " amo ", j'aime ! C'était trop facile ! Mais j'avais déjà appris quelque chose ailleurs, dans la rue. Quelque chose qui pouvait servir. Quand vous êtes dans la rue et que vous mourez de faim, vous apprenez à déchiffrer les visages. Il vous arrive de deviner qui va vous jeter quelques raisins et qui va vous donner un coup de pied. Quel monsieur dans la procession du saint sacrement va laisser tomber quelques gouttes de cire de sa bougie pour que vous puissiez la vendre. Et quel autre va vous dénoncer à la maréchaussée pour que vous soyez fouetté. Même les chiens, il vous arrive de distinguer entre ceux qui laisseront tomber l'os qu'ils ont dans la gueule et ceux qui vous mordront plutôt. C'est un apprentissage, tout un apprentissage des yeux, et que la faim rend bien plus efficace. Alors, ce que j'avais remarqué là dans la rue, j'ai commencé à le dessiner dans les marges de mes copies. Des visages. Aux grandes lettres majuscules, j'ajoutais un nez, des yeux, des oreilles, et sur les pages de musique, aux notes, des bras, des jambes. Je dessinais aussi sur les murs, les bancs, les portes. Les moines se fâchaient. Ils ont vu tout ça d'un bien mauvais œil, et ce fut pour moi un mauvais moment.

LE GUET : Ils ne vous ont pas chassé, au moins !

FILIPPO : C'est le prieur qui m'a sauvé. Au début. Plus tard, le prince. Les autres voulaient me chasser. Mais le prieur a dit non. Il leur a dit : Il ne sera pas copiste ? Tant pis, tant mieux. Il sera peintre! Il décorera notre chapelle. Il nous la rendra aussi belle que les autres. Nous aurons une chapelle aussi belle que celle des Dominicains. C'est ça qu'il voulait.

LE GUET : Alors, bien fait pour vous ! Et vous l'avez fait?

FILIPPO : Attendez. C'est un peu compliqué. Oui, je l'ai fait. Un mur entier. Des foules de gens, tous ceux qui viennent à l'église, des moines de tous les ordres, des bonnes femmes aussi, et puis un assassin, qui y arrive hors d'haleine, et la famille de sa victime, une fille enfin, qui donne à l'assassin, beau et barbu, une fleur. Et quand ils ont vu tout ça, les moines du couvent, ils ont crié : mais c'est la vie même! Cette fille-là, c'est la nièce du prieur, celle qui lui apporte les remèdes pour son asthme. Et puis ce garçon, c'est celui qui passe tous les jours dans la rue avec son chien. 
LE GUET : Nous aussi, nous le connaissons, ce garçon! Lui ou un autre. Mais quel triomphe! Pour vous.

FILIPPO : Oui, pendant cinq minutes. En attendant l'arrivée du prieur. Lui, y a mis fin.

LE GUET : Qu'est-ce qu'il a dit?

FILIPPO : Que ce n'étaient que des corps, des corps sans âme, que j'avais peints. Et qu'il ne fallait pas insister comme ça sur la chair. C'était l'âme qu'il voulait voir. L'âme!

LE GUET : Mais comment voir l'âme?

FILIPPO : Je ne sais pas. De la vapeur ? Ou peut-être l'image du nouveau-né qui sort de la bouche d'un mourant, vous savez, l'âme qui part. Il m'a dit: il y a Giotto, mon fils, comme modèle à suivre. Très bien. Je n'ai rien contre. Giotto, c'est merveilleux. Mais c'est d'une autre époque. Giotto nous aide à prier mais moi, je n'y aide pas. Moi, ma peinture, c'est trop de couleurs, trop de bras, trop de jambes. Pas de corps, m'a-t-il dit, l'âme! Mais il a bien aimé mon portrait de sa nièce. C'était une Salomé parfaite, dit-il. Celle qui a dansé, vous savez, peu vêtue. Mais ça n'a pas sauvé mon travail. Efface tout, m'a-t-il dit, efface tout et recommence.

LE GUET : Ce n'est pas vrai?

FILIPPO : Si. Et ça continue. Au couvent, c'est comme ça, toujours la même guerre. J'ai le prince de mon côté, heureusement pour moi, mais pas le prieur. J'ai essayé de le convaincre. Que l'âme sans corps, ce n'est pas possible. Voyez-vous, ce n'est pas en peignant mal qu'on arrive à faire de l'âme. Si vous mettez du jaune au lieu du blanc et du noir au lieu du jaune, c'est fort, peut-être, mais c'est laid, c'est inexact. Il faut faire les choses en ordre : pied gauche, pied droit, l'un après l'autre. La chair d'abord et l'âme ensuite. Prenez le visage le plus joli qui soit, celui de la nièce du prieur par exemple. Il n'est jamais si joli que vous ne puissiez y voir aussi la joie, la souffrance, la peur. L'émotion et la beauté sont parfaitement compatibles! Si ses yeux sont du bon bleu et bien dessinés, n'est-il pas facile d'y ajouter la touche qui donne la vie ? Et avec la vie, l'âme. Toutes les trois ensemble : la beauté, la vie et l'âme. Et même s'il n'y a que la beauté, mais la beauté sans âme, je ne l'ai jamais vue, mais même s'il n'y a que la beauté, n'avez-vous pas là ce que le bon Dieu a créé le mieux ? Et en le remerciant pour ça, vous allez trouver, à l'intérieur de vous-même, toute l'âme dont vous avez besoin. N'ai-je pas raison?

LE GUET : Sans doute, mon frère. La beauté, la vie et l'âme, c'est une jolie formule.

FILIPPO : Bien sûr. Je sais de quoi je parle. Mais le prieur, est-ce qu'il m'écoute ? Je ne suis pas un moine parfait, je le reconnais. Pour faire un moine parfait il ne faut pas prendre un gamin de huit ans qui crève de faim et le faire jurer qu'il n'embrassera jamais les filles! Maintenant, pourtant, je peins comme je veux. Je n'ai d'autre maître que moimême. Si, j'ai un maître: le prince, mais c'est plutôt un ami. Son palais, vous connaissez. J'y ai mes habitudes. Les avez-vous remarqués, ces grands anneaux, l'un sur l'autre, sur la façade? Ils servent à quoi selon vous? A attacher un cheval ? Un drapeau ? Pas uniquement, mes amis, pas uniquement !

Mais le prieur, il est toujours là. Même quand il n'est pas vraiment là, il est là, derrière mon épaule. J'entends sa voix : C'est de la décadence, mon fils, ce que tu es en train de faire. Ce n'est plus l'art pur et simple du passé. Tu ne seras jamais l'égal de Fra Angelico. Bon, d'accord, je ne serai jamais son égal! Je ne peux rien dire. Il est plus éduqué que moi, avec son latin, il devrait savoir. Alors, je fais un geste. Je fais quelque chose qui va leur plaire, à lui et aux autres. Je travaille, je travaille dur. Des saints, des saintes. Mais en fin de journée, un beau soir, beau et chaud comme celui-ci, et j'entends des bruits 
qui montent de la rue, quelqu'un qui rit, c'est plus fort que moi : ma coupe déborde, je me sauve, comme un gosse, je fais le fou. Voilà !

LE GUET : Et c'est comme ça que nous, nous vous avons trouvé dans la rue.

FILIPPO : Le vieux cheval, au pré après ses années de labeur, il lance les sabots dans l'air de la même manière. Et il n'a pas à écouter le meunier lui faire la leçon que manger de l'herbe ne sert pas à grand-chose! Qu'ils décident une bonne fois pour toutes ce qu'ils veulent ! C'est tout ce que je demande. Pas d'hypocrisie. Ils disent qu'ils aiment ce qu'en vérité ils n'aiment pas, et ce qu'ils disent qu'ils n'aiment pas, ils aiment! Vous comprenez ça, vous?

LE GUET : Mais non.

FILIPPO : Je suis trop honnête pour eux, trop simple. Je suis quelque part resté un enfant. Ce que je vois toujours devant moi c'est le jardin d'Eden et le bon Dieu en train de faire la femme pour l'homme. C'est la chair, qui sort de la main de Dieu. C'est tout ce que je vois, tout ce que je comprends. Et j'ai du mal à faire semblant de penser autrement.

(Un temps.)

Moi, je suis fini peut-être. Tiens, un peu de lumière dans le ciel. L'aube n'est pas loin. Mais il y en aura d'autres après moi. Nous avons justement un jeune qui vient chez nous, il veut étudier ma peinture. Il a l'air empoté. Les moines ne l'aiment pas trop, ils se moquent de lui mais lui, il s'en fiche. Il sait déjà tout ce que moi, je sais. Et il va aller plus loin encore. Après ma mort sans doute, je ne serai plus là pour le voir. Mais c'est inévitable. Lui ou un autre. Vous n'êtes pas de mon avis?

LE GUET : $\mathrm{Si}$, si.

FILIPPO : Vous me comprenez, vous. Mais ça me rend malade de n'être plus là pour les voir, ces nouveaux peintres qui vont nous dépasser. Parce qu'ils vont venir, c'est sûr. Et je ne serai plus là !

LE GUET : Faut pas le prendre comme ça, mon frère.

FILIPPO : C'est Dieu qui a fait ce monde, n'est-ce pas? Alors, pourquoi l'a-t-il fait? Et pourquoi l'a-t-il fait si beau, si merveilleux? Toutes sortes de choses, de formes, de couleurs, si différentes. Pourquoi ? Je vous pose une autre question. C'est une espèce de réponse. Est-ce que vous aimez votre ville? Notre belle ville de Florence. Oui, vous l'aimez, vous en êtes fiers. Avec sa rivière, les collines qui l'entourent, le beau ciel audessus. Mais si vous aimez tant ce qui n'est que bâtiments, ce qui n'est finalement que pierre et terre, eau et air, combien davantage vous devriez aimer ses habitants! Les hommes, les femmes, les enfants, qui sont sa vérité, la ville vivante. Et voici mon argument. Ne faut-il pas peindre ça ? La vie, la beauté de la vraie vie ?

LE GUET : Oui, sans doute, mais quand même, vous n'allez pas mettre des gens comme nous dans vos peintures!

FILIPPO : Je l'ai déjà fait ! Pas vous personnellement, mais des gens comme vous.

LE GUET : Mais à ce que j'ai compris, le prieur, les moines, ils n'ont pas aimé.

FILIPPO : Non.

LE GUET : Et je les comprends, mon frère. Il ne faut pas mettre n'importe quoi dans une peinture simplement parce que ça existe. La peinture est faite pour être belle, pour être noble.

FILIPPO : Attendez, voici un autre argument. Avons-nous besoin de la peinture? Si son but est de faire de la beauté, elle existe déjà, la beauté, sans elle. La beauté se trouve partout dans la nature, qui est la peinture de Dieu, si vous voulez. Est-ce que nous avons besoin d'une autre, la nôtre? 
LE GUET : Moi, je dirais oui, parce que peindre la beauté, c'est une façon de l'apprécier. Si on ne peignait pas la beauté, peut-être qu'on ne la remarquerait pas.

FILIPPO : La peinture nous aide à voir, oui, de ça je suis convaincu.

LE GUET : Elle nous aide à voir le meilleur côté des choses.

FILIPPO : Tu as raison. (À l'autre) Et ton visage à toi, mon vieux, dont j'ai dit qu'il ferait un parfait Judas, celui qui a fini pendu. Faut pas t'en offusquer! Parce que si je le peignais, il serait transformé. Pas en beauté peut-être mais tu aurais du plaisir à le contempler, ton portrait. Vous n'avez toujours pas un morceau de craie ou de fusain dans votre poche? Bon, une autre fois.

LE GUET : Vous avez un don précieux, mon frère, celui de créer la beauté.

FILIPPO : Créer, si seulement! C'est un trop grand mot. Au moins pour moi. Je préfère transformer, ou interpréter. Mais vous êtes gentils. Le prieur n'aimerait pas m'entendre parler d'interprétation. C'est son rôle à lui. C'est lui qui explique la parole de Dieu.

LE GUET : C'est autre chose.

FILIPPO : Oui, mais ça me fait plaisir de penser que nous travaillons ensemble, malgré lui. Pour lui, la peinture devrait dire aux gens : il faut aller à la messe. C'est tout. Mais il n'y a pas besoin de la peinture pour dire aux gens qu'ils doivent aller à la messe! Les mots du curé suffiront.

LE GUET : Normalement. Alors, si je vous ai bien compris, mon frère, votre idée, c'est qu'un bon tableau est une espèce de sermon, qu'il nous apprend des choses. Alors là, je suis d'accord avec vous.

FILIPPO : J'ai fait une fresque de St Laurent il y a six mois environ. Grandeur nature, même plus. Et quand j'avais fini et démonté l'échafaudage, j'ai demandé au frère à côté de moi : tu l'aimes? Beaucoup, m'a-t-il dit. Et un peu plus tard, il m'a raconté que les gens l'avaient déjà abîmée, la peinture. Ils se mettaient si en colère contre les bourreaux qui étaient en train de martyriser le saint qu'ils avaient abîmé la peinture avec leurs mains, avec des bâtons. Il en était ravi, le frère! Ils se sont attaqué si fort aux bourreaux qu'on voyait déjà les briques en dessous du plâtre. C'est ça, la peinture pieuse! C'est aussi bon qu'une prière. Et il m'a promis une autre commande pour l'an prochain. C'est bien, mais c'est quand même bête aussi !

(Un temps.)

C'est entre nous, tout ça. Je vous fais confiance. Ça doit être l'air du matin, si frais, si épicé, il m'enivre comme du vin, et je dis n'importe quoi. J'ai fait un peu trop l'école buissonnière mais je respecte l'Eglise. Et voici comment je vais me faire pardonner. C'est pour les bonnes sœurs. Ça va me prendre six mois. Vous pourrez alors aller le voir. $\mathrm{Au}$ couvent de St Ambroise. Qu'elles soient bénies, ces sœurs! Ce qu'elles veulent, c'est la Vierge Marie, l'enfant, et autour d'eux, des angelots comme une couronne de fleurs. De la douceur! Quelque chose de blanc, de si pur, de si léger, qu'on dirait un gâteau ! Et devant, quelques saints, bien sûr, debout ou à genoux. St Ambroise, leur patron, père et docteur de l'Eglise, St Jean Baptiste, qui protège la ville, et puis, de l'Ancien Testament, les souffrances de Job. Pourquoi les souffrances de Job? me demanderez-vous. C'est mon idée à moi. C'est parce qu'il nous représente, lui, nous les peintres, et toute la patience dont nous avons besoin. Et puis, ce n'est pas fini, parce que je vais ajouter, dans un coin, quelque chose de très inattendu. Devinez quoi... ? Moi-même!

LE GUET : Vous ? 
FILIPPO : Moi ! Le frère Filippo. Mais je sors du coin, pour entrer tout de même dans la lumière. Oh, en toute modestie! N'ayez pas crainte. Je sais très bien que je n'ai pas mérité de me trouver là, en telle compagnie. J'aurai ma robe de moine, la corde autour de la taille, mais ce n'est pas ça qui changera grand-chose. J'aurai l'air de quelqu'un qui veut se sauver, qui veut se cacher. Mais il y aura un ange qui s'avancera pour me prendre par la main, pour me mettre en présence des personnages divins, comme s'il leur disait : c'est quand même lui, tout imparfait qu'il soit, qui vous a créés! St Jean Baptiste, avec tous les poils de bêtes qu'il a autour de lui, est-il capable d'en faire un pinceau pour vous peindre? Non. Tout le monde sourit. Et moi, par modestie, je rougis, mais c'est vrai, c'est moi qui sais faire ce qu'ils ont envie de voir. Je suis ébloui par ma propre lumière. C'est comme si une centaine d'ailes battaient autour de moi. C'est trop fort ! Je dois vraiment me sauver.

C'est comme l'instant, vous savez, au milieu de vos plaisirs, où le mari rentre à l'improviste, et vous devez vous cacher dans un placard ou derrière un banc ! Mais je ne lâche pas la main de l'ange. Il ne me lâche pas non plus. Ou plutôt elle. Elle a le visage de la nièce du prieur. C'est Ste Lucie ! Et moi, comme ça, je me fais pardonner, et la chapelle sera enrichie d'un joli tableau. Allez le voir, dans six mois.

Messieurs, je dois vous quitter. Je vous serre la main.

LE GUET : On va vous accompagner, mon frère.

FILIPPO : Pas besoin, pas besoin, je vous remercie. Je connais mon chemin. En plus, c'est l'aube. Au plaisir!

Il sort d'un côté et le guet de l'autre.

\section{Andrea del Sarto (Robert Browning)}

(called 'the fautless painter')

But do not let us quarrel any more, No, my Lucrezia; bear with me for once: Sit down and all shall happen as you wish. You turn your face, but does it bring your heart? I'll work then for your friend's friend, never fear, Treat his own subject after his own way, Fix his own time, accept too his own price, And shut the money into this small hand When next it takes mine. Will it? Tenderly? Oh, I'll content him, - but tomorrow, Love! I often am much wearier than you think, This evening more than usual, and it seems As if - forgive now - should you let me sit Here by the window with your hand in mine And look a half hour forth on Fiesole, Both of one mind, as married people use, Quietly, quietly, the evening through, I might get up tomorrow to my work Cheerful and fresh as ever. Let us try. Tomorrow how you shall be glad for this! 
Your soft hand is a woman of itself,

And mine the man's bared breast she curls inside.

Don't count the time lost, either; you must serve

For each of the five pictures we require -

It saves a model. So! keep looking so -

My serpentining beauty, rounds on rounds!

- How could you ever prick those perfect ears,

Even to put the pearl there! Oh, so sweet -

My face, my moon, my everybody's moon,

Which everybody looks on and calls his,

And, I suppose, is looked on by in turn,

While she looks - no one's: very dear, no less!

\section{Andrea del Sarto (David Ball)}

Le jardin d'une maison près de Florence. Le soir. Un banc. La femme du peintre y est assise. Après un moment, il la rejoint.

ANDREA DEL SARTO: Lucrézia, un peu de patience pour une fois! Arrêtons de nous disputer. Tu permets? (Il s'assoit à côté d'elle.) Tu verras, les choses s'arrangeront. (Elle tourne la tête vers lui.) Ah oui, comme ça! C'est mieux, ton visage. Et ton cœur aussi ? Si je pouvais le voir... Je ferai ce travail pour l'ami de ton ami, et comme il le veut, n'aie pas peur. Le sujet sera traité selon ses idées. J'accepterai ses délais, son prix. Et l'argent, je le mettrai dans cette belle petite main que voici la prochaine fois qu'elle prendra la mienne. Avec tendresse, s'entend. Et il sera content, le monsieur, de mon travail. Mais demain, mon amour, demain. Je suis souvent bien plus fatigué que tu ne le penses, et ce soir plus encore que d'habitude. Mais il me semble, si j'ose le dire, que si tu me permettais de rester ici une demi-heure à tes côtés, je me lèverais demain matin de meilleur cœur. Ici, face à ce beau paysage, comme un vieux couple, la main dans la main, les mêmes idées dans la tête, tout tranquillement. Tu verras, demain, tu seras contente que nous ayons passé un tel moment ensemble.

Nos mains, voyons. La tienne, c'est toute la femme, sa douceur, comme la mienne, c'est le sein découvert de l'homme, où elle peut se blottir. Ce n'est pas du tout du temps perdu, tu sais, ce moment de repos. Pour toi aussi. Tu dois faire le modèle pour tous les cinq tableaux. C'est une économie! Oui, comme ça, cette même expression, c'est parfait! Tout est parfait, ta beauté ondulante. Tes oreilles, comment as-tu pu les faire percer? Même pour y mettre une perle. Ton visage est comme la lune, qui illumine le ciel de tout le monde, non seulement le mien. Chacun a sa lune à lui. Mais elle n'est à personne, bien sûr, la lune, tout comme ton visage. Ah, tu souris! C'est encore mieux. Mon tableau est déjà là, tout prêt. C'est l'harmonie, ce que nous, les peintres, cherchons avant tout. Tout baigne dans la même lumière. Maintenant, il y a au crépuscule une lumière argentée, comme au début, quand tu étais encore fière de moi. Non, ne dis rien pour l'instant. Tu sais que ce que je dis là, c'est la vérité. J'étais moi-même plein d'espoir. Mon art, ma jeunesse. Je n'avais pas encore appris l'art du compromis. De satisfaire le client avant tout.

Quel tableau enfin, n'est-ce pas, devant nous? Les arbres derrière le mur. Voilà la cloche du couvent qui sonne. Le dernier moine quitte le jardin. C'est l'automne. C'est l'automne partout, le crépuscule. De toute ma vie, la lumière meurt et part. Dans mes tableaux, c'est maintenant une lumière plus douce qui les inonde. Que veux-tu, nous sommes dans la main de Dieu. Il nous mène, il nous trompe peut-être. Nous nous 
croyons libres, et tout le temps nous sommes enchaînés. Mais c'est lui qui nous les impose, ces chaînes. Il faut les accepter. Et pourtant, tout cela est maintenant derrière nous. Tu ne le comprends pas, malheureusement, mon art.

LUCREZIA : Si. Au moins, j'entends ce que l'on dit de toi. Le légat, par exemple, la semaine dernière, a parlé de toi comme du maître qui savait tout faire à la perfection.

ANDREA : À la perfection! Oui, et même trop. C'est devenu tellement facile pour moi. Je n'ai plus besoin de faire d'étude préliminaire. Je suis envié, je sais. Il y a une vingtaine de peintres dans cette ville, rien qu'ici, qui rêvent de peindre comme moi. Le petit tableau que j'ai fait l'autre jour, et que tu as un peu barbouillé en y passant trop près dans ta robe bouffante, ils donneraient tout pour faire pareil. Ils n'y arrivent pas, eux, à la perfection. Tout le monde le dit. Mais en n'y arrivant pas, ils font peut-être mieux. Le moins, c'est parfois le plus! Il y a une lumière chez eux qui vient du ciel. L'humilité, la sincérité, l'effort désespéré, que sais-je, donnent une lumière plus divine. Ils montent plus haut que moi, ces peintres moins doués, même si ce qu'ils voient là-haut, ils n'arrivent pas à le faire voir. Mes œuvres montent, peut-être, moi pas.

Quel tapage on fait enfin, autour d'eux ! Et quel bruit ils font eux-mêmes! Qu'on dise du bien d'eux ou le contraire, c'est pareil, c'est toujours bouillonnant : ils sont si sensibles. Moi, pas du tout, je suis indifférent. Non, pas tout à fait quand même. Je sais ce que je vaux. Mais les critiques, les compliments, je les prends comme ça, sans émotion. C'est comme si on parlait d'un autre. Ça m'est égal, si quelqu'un dit de moi que le dessein, ou le coloris, est faux, exactement comme si quelqu'un d'autre disait qu'il est juste. On regarde la montagne, on parle d'elle, mais la montagne ne bouge pas, ne dit rien. Et pourtant, l'aspiration doit dépasser la réalisation. Autrement, pourquoi le ciel existe-til ?

La lumière qui inonde mes œuvres, c'est gris, un gris argenté, uniforme, qui crée au moins une ambiance de paix. Je sais ce que je suis capable de faire, les sommets que j'aurais pu atteindre, mais à quoi cela me sert-il ? Si je dis : moi et un autre, une double tête, nous aurions surpassé tout le monde. Sans doute, mais à quoi cela m'avance-t-il ? Tu vois ce tableau là ? C'est l'œuvre de ce jeune peintre d'Urbino qui est mort il y a cinq ans. Ou plutôt c'est une copie de son œuvre. C'est Giorgio Vasari qui me l'a envoyée. C'est merveilleux, n'est-ce pas ? Sauf que moi, je sais exactement comment il a procédé. C'était devant des papes, des rois, qu'il travaillait. Il est monté jusqu'au ciel, lui, plus haut que son art. Parce que là, je le vois bien, ce bras est mal dessiné. C'est à peine une faute, tellement l'âme de l'œuvre est pure et noble. Même un enfant peut s'en apercevoir. Tout de même, ce bras! Je pourrais le mettre comme il faut. Mais pour le reste, il me dépasse, je suis incapable de faire si bien. Mais pourquoi en suis-je incapable? Si toi, au début, si tu m'avais vraiment encouragé, m'avais poussé jusqu'aux sommets, toi et moi ensemble, nous aurions égalé Raphaël.

LUCREZIA : J'ai fait tout ce que tu m'as demandé de faire.

ANDREA : Oui, je sais, et même davantage. Plus que je méritais de toute façon. Mais si seulement, à ta beauté, beauté du front, des yeux, de la bouche, et à ta voix, si mélodieuse que je l'ai toujours suivie, comme l'oiseau trompé par le pipeau de l'oiseleur, si, à tout cela, tu avais seulement ajouté des idées ! Il y a des femmes qui ont des idées.

LUCREZIA : Et pourquoi pas moi? Pourquoi moi, je n'en aurais pas? Mais soyons honnêtes, Andrea. Si je t'avais dit : pense à Dieu, pense uniquement à lui et à la gloire. Oublie l'argent. Pense à l'avenir, à la notoriété, à être l'égal de Michel-Ange, de Raphaël, vous trois. Est-ce que tu l'aurais fait? Pour moi? 
ANDREA : Pour toi, peut-être. Plutôt que pour moi-même en tout cas. Mais tout est comme Dieu l'a de tout temps décidé. De toute manière, la force, l'ambition, le désir, doivent sortir de l'artiste lui-même. Il ne peut s'appuyer sur autrui. Je ne devrais pas avoir besoin de toi. Est-ce que Raphaël avait une femme? Ou Michel-Ange, est-ce qu'il en a une? Je constate que, dans ce monde, ceux qui peuvent ne veulent pas et que ceux qui veulent ne peuvent pas. Mais il y a de la volonté quelque part, de la force aussi, et c'est ainsi que nous, les moins que géants, nous arrivons quand même à quelque chose. Et à la fin, c'est Dieu qui punit ou qui récompense. Ça m'arrange, peut-être, que je sois un peu sous-estimé ici-bas, que je sois pauvre. Méprisé même. Tant et si bien qu'aujourd'hui par exemple, je n'osais pas sortir.

LUCREZIA : Pourquoi?

ANDREA : Les seigneurs français. Je n'avais pas envie de les croiser. Je préfère qu'ils passent à côté sans un mot. Mais parfois ils parlent. C'est dur. Mais que veux-tu, je dois le supporter. S'ils parlent, c'est qu'ils ont quelque chose à dire! De la part du roi François, et de toute cette année que j'ai passée à Fontainebleau... Ah, là, sous son regard, regard bienveillant. C'était de l'or qui brillait dans ses yeux ! Et je me sentais m'épanouir, comme si j'étais Raphaël. Il me mettait la main sur l'épaule, autour du cou, l'autre main enroulée dans sa barbe. Sa chaîne en or tintait tout près de mon oreille. Je n'en étais pas peu fier! Toute la cour autour de nous, le même regard que lui, franc, bienveillant, généreux, ils m'ont poussé vers le haut. J'ai travaillé pour eux mais aussi pour toi, ma belle. Ma meilleure récompense tout de même, ce visage souriant que voici ! Mais quelle année enfin, cette année française! Année royale! Si tu n'avais pas commencé, ici, à t'ennuyer...

LUCREZIA : Je m'inquiétais, c'était normal.

ANDREA : Bien sûr. Et c'était bien trop beau pour moi, une vie royale. Trop brillant. Je suis comme la chauve-souris, mes yeux préfèrent le noir. Mon monde à moi, c'est quand même ici, entre ces quatre murs. Alors, j'y suis rentré.

LUCREZIA : Tu me manquais! Tu ne vas quand même pas t'en plaindre?

ANDREA : Mais non, mais non. C'est bien ma plus belle réussite, n'est-ce pas, d'être aimé de toi? Une réussite que j'avais atteinte avant mes autres triomphes. Et si je suis toujours aimé de toi, qu'est-ce que j'ai perdu?

LUCREZIA : Mais rien.

ANDREA : Tu es à moi, Lucrézia ! Ta beauté, quel bonheur ! Les cheveux qui entourent ton visage, quelle auréole! Tu es ma Vierge Marie. On dit de celle de Raphaël qu'elle aide plus à la prière mais de la mienne : c'est quand même sa femme ! C'est, si tu veux, comme une excuse. Et vraiment, je crois, si on vous mettait tous les trois ensemble, toi, mon tableau et celui de Raphaël, on verrait que je n'ai pas démérité. Je vais te dire une chose. Un jour, c'est Michel-Ange en personne qui a dit à Raphaël : Tu sais, il y a un peintre à Florence, il ne paie pas de mine mais s'il était poussé et encouragé comme toi, entouré de papes, de rois, comme toi, alors, là, il te ferait courir ! Il a dit ça à Raphaël ! Et il avait raison. Le bras, là, ce n'est quand même pas correct ! Est-ce que j'ose.. ? Oui, ce n'est qu'un instant et il n'y a que toi pour me voir. Donne-moi le crayon-là. C'est comme ça que le bras doit être. Non, non, efface-le. Je ne peux pas. C'est quand même du Raphaël, l'âme de Raphaël ! Mais ces mots, c'est Michel-Ange qui les a prononcés ! LUCREZIA : Je sais, et j'en suis aussi fière que toi.

ANDREA : Encore, ton sourire! Tu sais, te faire plaisir, c'est tout ce que je désire. Et j'ai l'impression que si tu t'asseyais, comme ça, à mes côtés tous les soirs, je travaillerais mieux. Je gagnerais plus d'argent, pour toi. Voilà une étoile, dans le crépuscule, de plus 
en plus sombre maintenant. On a allumé les lampes, on entend les chouettes. Il faut bientôt rentrer. Dans notre maison, aujourd'hui si triste, qui était gaie au début. La justice de Dieu est implacable. Que le roi François me pardonne! J'ai bien reçu son argent, je l'ai bien dépensé. J'ai construit la maison avec. Et lui, il n'a pas reçu ses tableaux, pas ceux que je lui avais promis d'acheter.

LUCREZIA : Ce n'était pas tout à fait ta faute.

ANDREA : C'était la tienne, veux-tu dire ? Parfois je m'imagine que toutes ces pierres sont cimentées avec de l'or, avec de l'or français. Mais si tu m'aimes encore... (Un bruit.) C'est ton cousin?

LUCREZIA : Je dois y aller.

ANDREA : Il a toujours les mêmes besoins d'argent ? Il perd au jeu. Ça te fait sourire? Tu es trop gentille. Je resterai ici. Oisif, si tu veux. Je rêve. Je rêve que je suis de nouveau en France, à la cour du roi, en train de peindre, mon dernier tableau, le meilleur. Une Vierge Marie. Pas toi cette fois-ci. Un autre visage, pour que tu puisses l'admirer en toute sérénité. Pour que tu puisses entendre Michel-Ange dire ce qu'il vaudra, mon travail.

L'ami de ton ami, demain, je ferai tout ce qu'il veut. Ses idées, son portrait, tout. J'y ajouterai même, en petit cadeau, un autre tableau, pour qu'il ne pense pas à se plaindre. Et comme ça nous aurons assez d'argent pour que tu puisses payer les dettes de ton cousin. Pour que tu puisses t'offrir aussi quelque chose. Un beau foulard. Cela te fera plaisir ? Qu'est-ce qu'il a, ce cousin ? Que fait-il plus que moi pour te plaire ?

Pas de regrets. Je suis aussi apaisé qu'un vieil homme ce soir. Pas de regrets, ou si peu. Qu'est-ce que je changerais, de mon passé, si je pouvais ? Je ne peux pas le changer! Même le mauvais tour que j'ai joué au roi de France. Et mes parents, qui sont morts dans la misère. Mais est-ce que l'argent était uniquement à moi ? Pour devenir assez riche, qu'est-ce que j'aurais dû faire de plus, de pire? Tu vois? De toute façon, ils n'avaient rien connu d'autre, ayant vécu toute leur vie dans la misère. Et moi, j'ai parfois travaillé, et travaillé dur, sans être payé. Qu'un meilleur fils que moi peigne mes deux cents tableaux ! J'aimerais bien le voir.

Il y a peut-être un équilibre quelque part. Pour ce soir, ton affection me suffit. Il faut qu'elle me suffise. Que veux-tu, au ciel, peut-être, j'aurai enfin l'occasion, la dernière, la meilleure, de peindre là, si la cité céleste a bien quatre murs et que nous avons chacun son mur, nous quatre, Léonard, Raphaël, Michel-Ange et moi. Toi à mes côtés. Les trois autres sans femme, mais moi... Et ils me dépasseront toujours! Mais c'est mon choix, je t'ai choisie.

LUCREZIA : Mon cousin...

ANDREA : Oui, il t'appelle. Allez, mon amour, va le voir.

Elle sort. Lentement la nuit tombe. 